\title{
Fatty liver disease: the hepatic manifestation of metabolic syndrome
}

\author{
Amalia Gastaldelli \\ Hypertension Research (2010) 33, 546-547; doi:10.1038/hr.2010.60; published online 7 May 2010
}

$\mathrm{F}$ atty liver disease (FLD) refers to a spectrum of liver damage ranging from simple steatosis to nonalcoholic steatohepatitis, advanced fibrosis and cirrhosis. Obesity, a diet high in calories and fat, and a sedentary lifestyle are some of the risk factors for developing FLD. However, FLD is not only a liver disease. It is often associated with other systemic metabolic alterations, such as altered lipid profile, insulin resistance, general or preferential abdominal obesity and/or diabetes. ${ }^{1,2}$

The real prevalence of FLD is not known, but the perception is that its prevalence is increasing. ${ }^{3}$ In the general population, it is estimated that $20-45 \%$ of people have liver steatosis. The prevalence increases to $57 \%$ in obese subjects, $70 \%$ in diabetic subjects and $90 \%$ in morbidly obese people. The data on the incidence of FLD are still scarce. ${ }^{3}$ In this context, the paper by Tsuneto et al. ${ }^{4}$ published in this issue of Hypertension Research is very important. The authors evaluated the incidence of FLD in 1635 Nagasaki atomic bomb survivors who were followed for a mean of 12 years: they showed an incidence of 19.9 per 1000 people per year. Despite the limitation of the study cohort, namely, the subjects are all atomic bomb survivors and thus the results cannot be translated to the general population, the data are quite similar to those published by Bedogni et al. ${ }^{5}$ for the Italian population examined in the Dionysos study, which reported an incidence of 18.5 per 1000 people per year.

Dr A Gastaldelli is at the Consiglio Nazionale delle Ricerche (CNR) of Clinical Physiology, Via Moruzzi 1, Pisa 56126, Italy.

E-mail: amalia@ifc.cnr.it
Tsuneto et al. found that age, obesity (body mass index (BMI) $\geqslant 25 \mathrm{~kg} \mathrm{~m}^{-2}$ ), hypertriglyceridemia and, to a lesser extent, hyperten$\operatorname{sion}^{4}$ were among the variables that predicted development of FLD. Glucose intolerance status was found to be associated with an increased risk of developing FLD, but the significance was lost when the data were corrected for age. Another important result found by Tsuneto et al. was that BMI and serum triglyceride levels increased steadily during the mean 12-year study period in subjects who developed FLD, ${ }^{4}$ whereas, in the other group, BMI and triglyceride concentrations remained constant. This finding is in agreement with previous results showing a strong positive correlation between BMI and visceral and intrahepatic fat accumulation in subjects with FLD. ${ }^{2,6}$ Moreover, hyperglycemia is a risk condition for FLD, independent of obesity: patients with diabetes have a greater accumulation of intrahepatic and visceral fat despite similar BMIs. ${ }^{2,6}$

FLD is often considered to be the hepatic component of metabolic syndrome. ${ }^{7}$ Metabolic syndrome is a cluster of metabolic and cardiovascular risk factors that predicts diabetes and cardiovascular disease (CVD) better than do any of its individual components. ${ }^{8}$ The latest joint statement of the International Diabetes Federation and the American Heart Association/National Heart, Lung, and Blood Institute qualifies a person as having metabolic syndrome if he or she has at least three abnormal findings from among the following: abdominal obesity as defined by an increased waist circumference (population and country specific), low high-density lipoprotein (HDL) cholesterol, elevated blood pressure, elevated fasting glucose or elevated triglyceride con- centration. ${ }^{8}$ FLD and increased levels of hepatic enzymes (i.e., alanine aminotransferase and $\gamma$-glutamyl transpeptidase, often used as markers of FLD) are used to predict the onset of metabolic syndrome, incident CVD, and even death. $6,9,10$

Liver fat is highly correlated with all the components of metabolic syndrome, independent of obesity. In subjects with FLD, hepatic overproduction of glucose, verylow-density lipoproteins (VLDLs), C-reactive protein (CRP) and coagulation factors increase the risk of type 2 diabetes and cardiovascular disease. ${ }^{1,6,10}$ Hyperglycemia induces a series of alterations, including endothelial dysfunction, cellular proliferation, changes in extracellular matrix conformation and impairment of LDL receptor-mediated uptake, decreasing the in vivo clearance of LDL. Small dense LDLs associated with high circulating VLDLs have a higher affinity for intimal proteoglycans, leading to the penetration of more LDL particles into the arterial wall. CRP can also accelerate atherosclerosis by increasing the expression of plasminogen activator inhibitor-1 and adhesion molecules in endothelial cells, inhibiting nitric oxide formation and increasing LDL uptake into macrophages. ${ }^{1,6,10}$ All these metabolic abnormalities have been shown to directly or indirectly promote atherosclerosis; in fact, carotid intima-media thickness has been found to be increased in subjects with FLD. ${ }^{10,11}$

Despite all the evidence, FLD or increased hepatic enzymes are not considered among the criteria indicating metabolic syndrome. ${ }^{8}$ The paper by Tsuneto et al. clearly shows that subjects who developed FLD had high blood pressure, low HDL cholesterol, and a constant increase in triglyceride concentrations and 
BMI throughout the study period (waist was not measured and fasting glucose was not reported). ${ }^{4}$ These longitudinal data are therefore very important for the future inclusion of FLD among the criteria for metabolic syndrome.

1 Cusi K. Role of insulin resistance and lipotoxicity in non-alcoholic steatohepatitis. Clin Liver Dis 2009; 13 : 545-563.

2 Gastaldelli A, Cusi K, Pettiti M, Hardies J, Miyazaki Y, Berria R, Buzzigoli E, Sironi AM, Cersosimo E, Ferrannini E, Defronzo RA. Relationship between hepatic visceral fat and hepatic insulin resistance in nondiabetic and type 2 diabetic subjects. Gastroenterology 2007; 133: 496-506.
3 Ong JP, Younossi ZM. Epidemiology and natural history of NAFLD and NASH. Clin Liver Dis 2007; 11: $1-16$.

4 Tsuneto A, Hida A, Sera N, Imaizumi M, Ichimaru S, Nakashima E, Seto S, Maemura K, Akahoshi M. Fatty liver incidence and predictive variables. Hypertens Res 2010; 33: 638-643.

5 Bedogni G, Miglioli L, Masutti F, Castiglione A, Croce LS, Tiribelli C, Bellentani S. Incidence and natural course of fatty liver in the general population: the Dionysos study. Hepatology 2007; 46: 1387-1391.

6 Vanni E, Bugianesi E, Kotronen A, De Minicis S, YkiJarvinen $H$, Svegliati-Baroni $G$. From the metabolic syndrome to NAFLD or vice versa? Dig Liver Dis 2010; 4: 320-330.

7 Marchesini G, Brizi M, Bianchi G, Tomassetti S, Bugianesi E, Lenzi M, McCullough AJ, Natale S, Forlani G, Melchionda N. Nonalcoholic fatty liver disease: a feature of the metabolic syndrome. Diabetes 2001; 50 : 1844-1850.

8 Alberti KG, Eckel RH, Grundy SM, Zimmet PZ, Cleeman JI, Donato KA, Fruchart JC, James WP, Loria CM,
Smith Jr SC. Harmonizing the metabolic syndrome: a joint interim statement of the International Diabetes Federation Task Force on Epidemiology and Prevention; National Heart, Lung, and Blood Institute; American Heart Association; World Heart Federation; International Atherosclerosis Society; and International Association for the Study of Obesity. Circulation 2009; 120: 1640-1645.

9 Lee DS, Evans JC, Robins SJ, Wilson PW, Albano I, Fox CS, Wang TJ, Benjamin EJ, D'Agostino RB, Vasan RS. Gamma glutamyl transferase and metabolic syndrome, cardiovascular disease, and mortality risk: the Framingham Heart Study. Arterioscler Thromb Vasc Biol 2007; 27: 127-133.

10 Targher G. Non-alcoholic fatty liver disease, the metabolic syndrome and the risk of cardiovascular disease: the plot thickens. Diabet Med 2007; 24: 1-6.

11 Gastaldelli A, Kozakova M, Hojlund K, Flyvbjerg A Favuzzi A, Mitrakou A, Balkau B. Fatty liver is associated with insulin resistance, risk of coronary heart disease, and early atherosclerosis in a large European population. Hepatology 2009; 49: 1537-1544. 